\title{
Sobre el anti-realismo de Wittgenstein y su aplicación al programa
} chomskiano*

\author{
On Wittgenstein's Anti-realism and its Application to the Chomskyan Program
}

Sergio Mota ${ }^{\dagger *}$

\begin{abstract}
Resumen
El objetivo principal de este trabajo es mostrar un mapa conceptual en el que situar a Wittgenstein dentro de las distintas concepciones acerca de la lógica y la matemática. Después analizaré cómo se puede aplicar su concepción de la matemática al formalismo. Por último, analizaré el procedimiento mecánico finito definido por Chomsky y su relación con las nociones de mente y de representación (mental) dentro del marco conceptual expuesto previamente.
\end{abstract}

Palabras clave: anti-realismo - Wittgenstein - Chomsky - representación mental - mente

\begin{abstract}
The main goal of this paper is to present a conceptual framework in which we can situate Wittgenstein's views and compare them with different conceptions about the nature of Logic and Mathematics. Then I will consider how his conception of Mathematics can be applied to the formalism. Finally, I will analyze the finite, mechanical procedure defined by Chomsky and its relation to the notions of mind and (mental) representation, within the conceptual framework previously exposed.
\end{abstract}

Keywords: anti-realism - Wittgenstein - Chomsky - mental representation - mind

\footnotetext{
* Recibido: 3 de Febrero de 2014. Aceptado en versión revisada: 13 de Marzo de 2014.

+ Departamento de Psicología Básica, Universidad Autónoma de Madrid, España. Para contactar al autor, por favor escriba a: sergio.mota.v@gmail.com.

* Mi más sincero agradecimiento a los profesores Anastasio Alemán y Noam Chomsky por su inestimable ayuda y disponibilidad en las discusiones de algunos de los puntos analizados en el presente artículo. Asimismo mi gratitud al profesor Carlos U. Moulines por su lectura pormenorizada y útiles comentarios a una versión previa.

Metatheoria 4(2)(2014): 35-51. ISSN 1853-2322.

(c) Editorial de la Universidad Nacional de Tres de Febrero. Publicado en la República Argentina.
} 
A lo largo de la historia se han propuesto diferentes concepciones acerca de la naturaleza de la lógica y la matemática. El análisis que voy a presentar aquí le debe mucho al que ha presentado Alemán (2011) con anterioridad. Fundamentalmente, voy a seguir las líneas maestras por él esbozadas incluyendo algunas concepciones que no aborda directamente. En su libro Lógica, matemáticas y realidad (Alemán 2011) recoge una distinción general entre las diferentes concepciones de la lógica y la matemática y su relación con la realidad. Así, sugiere una primera división entre aquellas concepciones que son descriptivistas y aquellas que son no-descriptivistas.

Las descriptivistas hacen referencia a aquellas concepciones que entienden las expresiones lógicomatemáticas como descripciones de la realidad. Sostienen, por tanto, que tales expresiones dicen algo acerca de algún aspecto de la realidad que describen. De qué realidad hablemos nos lleva a una subdivisión: Platonismo y Empirismo. En mi opinión, en esta categoría habría que destacar también el Logicismo.

El Platonismo postula que la realidad que es descrita por los enunciados lógicos y matemáticos es una realidad ideal, abstracta, fuera de los límites espacio-temporales. Un autor de referencia es Gödel (véase, por ejemplo, Gödel 1944).

Frente al Platonismo encontramos el Empirismo. Para éste último, los enunciados lógicomatemáticos describen una realidad espacio-temporal, perceptible por los sentidos, siendo innecesario, por tanto, apelar a un tercer reino platónico. Dentro de esta concepción empirista, alternativa al empirismo lógico, encontramos a Maddy, Kitcher o Tymoczko, entre otros (recomiendo Alemán 2011, capítulos 1 y 2 para más detalles y referencias).

He propuesto más arriba el Logicismo como una concepción de la lógica y la matemática que cae dentro de los descriptivistas. ${ }^{1}$ Para los logicistas, el aspecto fundamental es que creen que la matemática puede reducirse a la lógica. Sin embargo, logicistas reconocidos como Frege y Russell parecen sostener la existencia real de los objetos lógico matemáticos apelando a la posibilidad de usar variables ligadas para referirse indistintamente a entidades abstractas independientes de la mente y, por tanto, no construidas o inventadas; quizá descubiertas o, como dice Frege, captadas.

En relación con las concepciones no-descriptivistas, las cuales niegan que los enunciados lógicomatemáticos describan la realidad, esto es, nieguen o aseveren nada, se encuentran los constructivistas. Podríamos decir que dentro esta categoría se encuentran los Intuicionistas, Formalistas y Convencionalistas. Así, los primeros (al igual que el conceptualismo) sostienen que las construcciones lógico-matemáticas son, en todo caso, productos del ejercicio intelectual, construcciones. Es decir, los objetos lógico-matemáticos no se descubren, sino que se inventan. Para una concisa presentación del Intuicionismo véase Kleene (1952, pp. 46-53).

Por su parte, los formalistas, encabezados por Hilbert, consideran, en alguna medida, insatisfactorios tanto el logicismo como el intuicionismo. Quine (1980, p. 15), argumenta tal insatisfacción apelando a dos razonamientos opuestos. En el primero de ellos, concede que formalismo (relacionado con el nominalismo) e intuicionismo atacan conjuntamente el recurso de los logicistas de los universales (entidades abstractas que son descubiertas e independientes de la mente). En el segundo de ellos, el formalista se pone del lado de los logicistas y ambos atacan conjuntamente a los intuicionistas al oponerse a las restricciones específicas de la matemática intuicionista. Para una presentación del Formalismo, recomiendo Kleene (1952, pp. 53-65).

\footnotetext{
1 En una comunicación personal con el profesor Moulines, me indicó que el logicismo, en efecto, no tiene por qué ser considerado como una forma de realismo. "En sí mismo considerado, el logicismo sólo sostiene que las matemáticas son reducibles a la lógica", y añade, "otra cuestión es que históricamente muchos logicistas hayan adoptado adicionalmente una posición realista". Considero esta caracterización correcta, y aquí, siguiendo a Quine (1980), hago referencia a logicistas como Frege y Russell, los cuales parecen haber adoptado una posición realista.
} 
El Formalismo es, tal como indica Alemán (2011, p. 19), el antecedente histórico del Convencionalismo y se aprecian ciertamente semejanzas entre ambas concepciones. Así, los convencionalistas tampoco encuentran razones suficientes para desestimar los sistemas formales de la matemática clásica construidos de manera diferente a como lo hace la matemática intuicionista. De este modo, se aceptan diferentes sistemas formales definidos con base en diferentes reglas para el manejo de los signos lógico-matemáticos; se concibe como un juego de notaciones formales. Dicho de otro modo, las expresiones o enunciados de la lógica y de la matemática son reglas, reglas gramaticales, como diría Wittgenstein. De este modo, se pueden investigar y, de hecho, esto ya se ha realizado, propiedades como la "completud" o la "decidibilidad" (véase Gödel 1931, Church 1936, Turing 1937, Post 1943). Finalmente, es interesante apuntar que aunque la filosofía de la matemática de Wittgenstein se sitúe dentro del convencionalismo, este autor no pareció interesarse por los fundamentos de la matemática, como sí hicieron los formalistas, algo que a mi juicio es relevante (véase Monk 1990).

En la siguiente sección, desarrollaré en mayor detalle la posición anti-realista de Wittgenstein a lo largo de su obra. En la tercera sección discutiré bajo el prisma wittgensteiniano algunas de las propuestas más importantes dentro de la concepción formalista, proponiendo que todos los sistemas formales son objetos lógico-matemáticos construidos y, por tanto, no aluden a entidades independientes descubiertas por el lógico o el matemático. En la cuarta sección abordaré algunas de las implicaciones del análisis previo para la discusión sobre la naturaleza de lo mental, fundamentalmente dentro del programa chomskiano. Finalmente, presentaré las conclusiones.

En esta sección voy a presentar el anti-realismo que caracterizó la obra de Wittgenstein, desde la aparición del Tractatus Logico-Philosophicus (1922), pasando por las Philosophical Remarks ([1975a] 1997) y Philosophical Grammar ([1974] 1992), hasta llegar a las Philosophical investigations (1958), centrándose en esta última etapa, sobre todo, en la noción de regla gramatical. La idea de que la matemática está constituida por reglas gramaticales también es fundamental en sus Remarks on the Foundations of Mathematics (1978). Asimismo, defenderé que la lógica y la matemática no describen hechos, sino que son sistemas construidos, creados o inventados (uso estos términos de manera intercambiable).

En lo referente al Tractatus, hay diferentes interpretaciones sobre el anti-realismo de Wittgenstein, alternativa a la interpretación empirista que de tal obra intentaron establecer los positivistas. Así, Pinto (2002) defiende el anti-platonismo presente en el Tractatus, aunque a mi juicio, los ejemplos que expone para ello no parecen ser los que mejor muestran tal posición, y en todo caso no son los únicos. Así, Pinto (2002) fundamenta el anti-platonismo en la filosofía de la matemática del Tractatus apelando a la siguiente proposición 6.211, que dice:

[E]n la vida, una proposición matemática no es jamás lo que necesitamos; más bien utilizamos las proposiciones matemáticas sólo para inferir, de proposiciones que no pertenecen a la matemática, otras que tampoco pertenecen a la matemática (cursivas en el original, Pinto 2002, p. 138).

Sin embargo, esta proposición no nos permite establecer por sí misma el anti-platonismo, ya que bien podría servir para establecer una posición anti-empirista de la matemática. Esa proposición quiere decir que utilizamos las reglas matemáticas para inferir de los enunciados 'tengo dos piedras' y 'tengo dos piedras' el enunciado 'tengo cuatro piedras'. Enunciados empíricos, en todo caso. Y es que esta postura anti-descriptivista en el Tractatus se refleja en que "las proposiciones de la matemática no expresan pensamiento alguno” (Pinto 2002, proposición 6.21, p. 138).

Una muestra más acertada, en mi opinión, del anti-platonismo del Tractatus, se evidencia en la proposición 6.232: "Frege dice que ambas expresiones [entiéndase ' $1+1+1+1$ ' y ' $(1+1)+(1+1)$ '] tienen la misma referencia, pero diferente sentido" y continúa "[p]ero lo esencial de una ecuación es que no es necesaria para mostrar que las dos expresiones que combina el signo de igualdad tienen el mismo 
significado, pues esto puede desprenderse de las dos expresiones mismas”. Es decir, Wittgenstein está negando que expresiones como ' $1+1+1+1$ ' tengan una referencia, una entidad ideal a la que se refiere o que denota. Al mismo tiempo, está diciendo que tales expresiones no dicen que tengan el mismo significado, sino que eso se muestra en los signos; la ecuación sería la regla explícita que muestra tal igualdad.

En lo referente al dominio de la lógica, Frege sostuvo que la referencia de una función es su valor de verdad, esto es: lo Verdadero o lo Falso (Frege 1892). Esta concepción es también atacada por Wittgenstein en el Tractatus. "Mi idea fundamental es que las constantes lógicas no actúan como representantes de nada" (4.0312); esto es, no son nombres cuyas referencias sean lo Verdadero o lo Falso. Esto es especialmente relevante en el caso de tautologías -o, siguiendo a Wittgenstein, "las proposiciones de la lógica" (6.1) - y contradicciones, pues ambas carecen de sentido, no expresan pensamiento alguno.

Por otro lado, como muy bien ha señalado Ruíz Abánades (2009), los nombres propios empleados en el Tractatus, en un sentido lógico, no son, como asumieron los positivistas, términos observacionales referentes a propiedades observables. Podemos decir, por tanto, que los nombres en este sentido lógico (formal) del Tractatus no representan propiedades observacionales. Éste párrafo y el anterior muestran claramente el anti-realismo expuesto por Wittgenstein en el Tractatus.

Con respecto a la noción de objeto (lógico o matemático) en el Tractatus, Wittgenstein muestra el uso que le da dentro de una concepción no-descriptivista (y no sólo anti-platonista). En 4.1272, Wittgenstein nos indica que "el nombre variable ' $\mathrm{x}$ ' es el signo propiamente dicho del pseudoconcepto [o concepto formal] objeto" (en cursivas en el original). Y continúa "[s]iempre que la palabra 'objeto'... se usa correctamente, se expresa en las notaciones conceptuales mediante un nombre variable”; así, pone como ejemplo que la proposición "Hay dos objetos tales que..." se expresa en tal notación como " $(\exists x, y) \ldots . .$. . "De este modo uno no podría decir, por ejemplo, 'Hay objetos' del mismo modo en que podría decir "Hay libros", por la sencilla razón de que lo primero no es una cuestión acerca de lo que hay, sino, sencillamente, conceptual. Nos dice Wittgenstein: "[l]a pregunta por la existencia de un concepto formal es un sinsentido. Pues ninguna proposición puede responder a tal pregunta" (4.1274), dado que no es una cuestión consistente en comparar una proposición con la realidad (empírica o platónica). Los objetos son, sencillamente, construcciones que pertenecen al simbolismo y por ello, ya se muestran en la notación.

Unida a la noción de objeto está, como he mostrado, la de concepto formal. En 4.12721, Wittgenstein indica que "[u]n concepto formal está ya dado en cuanto se da un objeto que cae bajo él" (cf. 4.126; 4.127; 4.1271). Conceptos formales (variables) son, por ejemplo, la forma general o término general de una serie de formas (cf. 4.1273). Tal forma general (o variable) queda expresada así: [a, $x$, O'x]. ${ }^{2}$ Tal expresión muestra una regla para definir series de formas y puede ser expresada por un sistema de ecuaciones recursivas (Mota 2013, 2014): ${ }^{3}$

$$
\begin{aligned}
& \left.\mathrm{O}^{(0)}\right)^{\prime}(a)=a, \\
& \mathrm{O}^{(n)}(a)=\mathrm{O}^{\prime} \mathrm{O}^{(n-1) \prime}(a) .
\end{aligned}
$$

La recursión caracteriza la definición de cada término y, por tanto, de la serie como un todo (esto se corresponde con el nivel de análisis referente a qué hace un procedimiento tal; véase Mota 2013, 2014), mientras que la iteración caracteriza cómo se procede para generar cada término; a saber,

\footnotetext{
2 En 5.2522, explica en qué consiste esta notación. Así, "[e]l primer término...es el comienzo de la serie de formas, el segundo es la forma de un término $x$ cualquiera de la serie y el tercero la forma de aquel término de la serie que sigue inmediatamente a $x$ ".

3 Por 'recursión' hay que entender el término definido dentro de la Lógica Matemática, y más concretamente, dentro de una rama especializada de ésta, la teoría de la computabilidad. Siguiendo a Soare $(1996,2009)$, 'recursión' se predica del método o reglas para definir funciones y predicados. Así, una función está definida en sentido técnico por recursión cuando para definirla para un argumento $x$ hacemos uso de sus propios valores previamente computados para argumentos menores que $x$; pudiendo emplearse también funciones previamente definidas (cf. Cutland 1980, Kleene 1943). Este es el significado original y primario de 'recursión' (véase Soare 1996, 2009, para más detalles).
} 
aplicando en sucesión la operación O' sobre el último valor generado, lo que permite generar series como a, O'a, O'O'a,... y así sucesivamente (Mota 2013, 2014).

Lo mismo vale para los términos generales de una proposición ' $[\tilde{p}, \tilde{\xi}, \mathrm{N}(\tilde{\xi})]$ ' y de un número natural ' $\left[\Omega^{0} x, \Omega^{v-1} x, \Omega^{v} x\right]=[0, \xi, \xi+1]$ '.

La definición recursiva del procedimiento mecánico finito que nos proporciona Wittgenstein para generar toda proposición (o función de verdad) y decidir en un número finito de pasos si es o no una tautología es como sigue (Mota 2013, 2014):

$$
\begin{aligned}
& N^{(0)},(\tilde{\xi})=\tilde{\xi} \text { Def., } \\
& N^{(n)}(\tilde{\xi})=N^{\prime} N^{(n-1)},(\tilde{\xi}) \text { Def. }
\end{aligned}
$$

Como el propio Wittgenstein señala en 5.502, “ $N(\tilde{\xi})$ es la negación de todos los valores de la variable proposicional $\xi$ ". Por tanto, como indica en 5.51 , si $\xi=p$, entonces $N(\tilde{\xi})=($ FVFV) $(p)$. Por otra parte, si $\xi=p, q$, entonces $N(\tilde{\xi})=($ FFFV $)(p, q)$. Así, la operación $N(\tilde{\xi})$ es equivalente a la operación de la negación conjunta, la cual se representa por medio de la barra de Sheffer (Mounce 1981). El procedimiento que arriba defino puede generar proposiciones como las que Pinto (2002, p. 142) propone como ejemplo: [(p,q); $(\xi, \zeta) ; N(N(N(\xi, \xi), \zeta), N(N(\xi, \xi), \zeta))]$. Una expresión tal se puede simplificar poniendo ' $N(p, q)$ ' en lugar de ' $N(N(\xi, \xi), \zeta)$ ', en donde ' $N\left(\xi\right.$, $\xi$ )' está por ' $p_{(\mathrm{FVFV})}$ ' y ' $\zeta$ ' está por ' $q_{(\mathrm{VVFF})}$ '. Con esto tenemos ' $N[N(p, q), N(p, q)]$ ' que equivale a ' $N\left(N\left(p_{(\mathrm{FVFV})}, q_{(\mathrm{VVFF})}\right)\right)$ ', puesto que es la negación conjunta de ' $N\left(p_{(\mathrm{FVFV})}, q_{(\mathrm{VVFF})}\right)$ ' consigo misma. Una definición recursiva de tal expresión es: $N^{(2) '}(p, q)=N^{\prime} N^{(1)}$ ' $(p, q)$. En el caso que acabo de analizar, sería suficiente poner '(VVFV) $(p, q)$ ' y el procedimiento mecánico finito arriba definido permite generar (y decidir en un número finito de pasos) otras proposiciones a partir de ella (cf. 6.002). Esto muestra, por otro lado, el finitismo que, en general, caracteriza la obra de Wittgenstein.

De hecho sería posible establecer el siguiente esquema, que completa a 5.101:

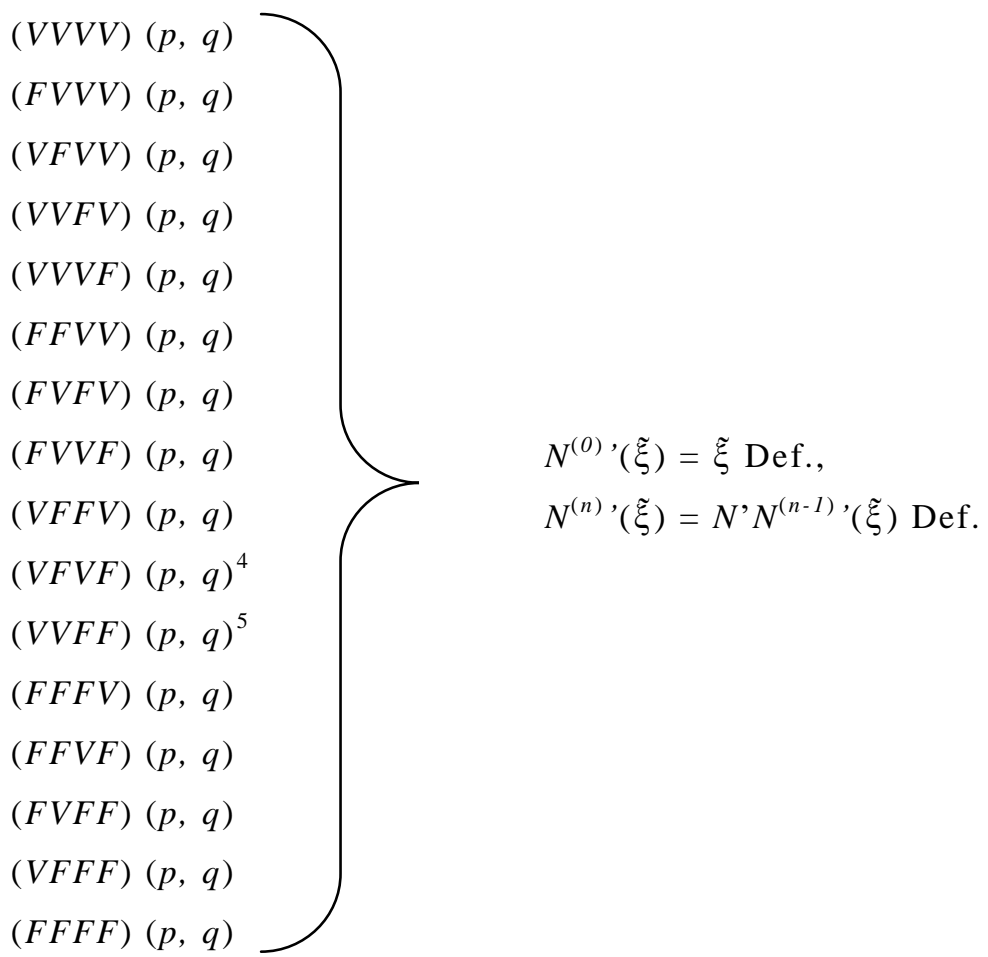

Este esquema muestra con claridad que (I) todas "[l]as funciones de verdad pueden ordenarse en series" (5.1), y (II) que la lógica proposicional es decidible, completa y consistente. Así, tenemos que tanto

\footnotetext{
4 Tal como indica Wittgenstein en 5.101, este signo proposicional está por $p$.

5 En este caso, está por $q$.
} 
dicha serie como la de los números naturales son objetos formales que instancian el concepto formal de forma general de una serie de formas. Con esto, queda expuesta y analizada la perspectiva antidescriptivista de Wittgenstein respecto al Tractatus. Veamos ahora su posición anti-descriptivista en etapas posteriores.

Pinto (2002, p. 139, nota al pie 4), argumenta que las reglas lingüísticas son, en primer lugar, regularidades empíricas que un intérprete discierne en el comportamiento de un hablante; en segundo lugar son generalizaciones universales; y, en tercer lugar, regularidades normativas. Me centraré en el primer sentido propuesto, puesto que, en mi opinión, puede crear confusión. Creo que en este punto habría que diferenciar dos tipos de enunciados: ${ }^{6}$

(a) Una semana está compuesta de siete días.

(b) 'Una semana está compuesta de siete días' expresa una regla semántica del castellano.

El enunciado (a) expresa una regla que define un sistema conceptual (un juego, en el sentido empleado por Wittgenstein), y en este caso expresa una regla lingüística. Pero un enunciado como (b) es un enunciado empírico, el cual no expresa una regla. Las reglas no son susceptibles de verificación o falsación, mientras que un enunciado como (b) sí lo es, pues hay que atender al comportamiento de los hablantes de una comunidad para averiguar si la siguen o no. Pinto, al decir que las reglas son regularidades empíricas, parece estar confundiendo enunciados del tipo (a) con enunciados del tipo (b), aunque no ponga ningún ejemplo concreto para ilustrar su posición. Es evidente que una regla gramatical como (a) expresa una definición, mientras que (b) es un enunciado sobre una conducta. ${ }^{7}$

En cualquier caso, Wittgenstein es muy claro al respecto: las reglas gramaticales no rinden cuentas ante ninguna realidad, sea empírica o platónica, algo que deja muy claro en sus obras "intermedias" y "posteriores". Así, en Philosophical Investigations (1969), encontramos que Wittgenstein analiza el papel de las reglas gramaticales para el uso de 'no':

No tiene sentido discutir si estas u otras reglas son las correctas para la palabra 'no' (quiero decir, si son adecuadas a su significado). Pues la palabra no tiene aún ningún significado sin estas reglas; y si cambiamos las reglas, obtiene otro significado (o ninguno) y en tal caso podríamos también cambiar la palabra. (Wittgenstein 1958, p. 351)

Veamos, pues, con más detalle, por qué no tiene sentido plantear esta discusión con respecto a las reglas gramaticales. Para ello, hay que acudir a dos obras fundamentales para entender esta noción, a saber: las Observaciones filosóficas y la Gramática filosófica. En las Observaciones filosóficas, Wittgenstein establece que:

Yo no llamo a una regla de representación una convención si puede quedar justificada en proposiciones, las cuales describen lo que es representado y muestran que la representación es adecuada. Las convenciones de la gramática no permiten que se les justifique mediante la descripción de lo que es representado. Toda descripción así presupone ya las reglas de la gramática (Wittgenstein [1975a] 1997, § 7, el énfasis es mío).

Es evidente que las reglas de uso de la palabra 'no' son convencionales en este sentido que emplea Wittgenstein. Pero aún hay más. En Philosophical Grammar Wittgenstein argumenta lo siguiente:

La gramática no tiene que rendirle cuentas a ninguna realidad. Las reglas gramaticales determinan el significado (lo constituyen) y, de esa manera, no son responsables [no dependen] de ningún significado siendo también, en esa medida, arbitrarias (Wittgenstein [1974] 1992, § 133).

\footnotetext{
${ }^{6}$ Agradezco al profesor Alemán sus inteligentes observaciones en este punto mediante correspondencia personal.

${ }^{7}$ Decir, por ejemplo, que el enunciado (a) no es una regla que se siga en la comunidad X, no es falsar empíricamente la regla; del mismo modo que si se sigue no se ha confirmado empíricamente. La razón es simple, no podemos decir que la hemos confirmado porque sin esa regla 'semana' no tendría aún significado. Luego si no es confirmable empíricamente tampoco es falsable. Por tanto, descubrir que se sigue una regla (enunciado (b)) no es lo mismo que la definición de la regla (enunciado (a)) y por ello no es correcto definir una regla gramatical como regularidad empírica.
} 
Esto es, no se puede apelar al significado de una palabra para justificar (y determinar) si una regla gramatical es adecuada o correcta para dicho significado pues sin esa regla la palabra no tiene aún significado; y es, precisamente, el hecho de que las reglas sean arbitrarias lo que va en contra de la posibilidad de su justificación (Wittgenstein [1974] 1992, § 133). Es decir, una regla no es gramatical si puede ser justificada "por el hecho de que una representación acorde con ella coincida con la realidad" (Wittgenstein [1974] 1992, §134). Por tanto, un criterio claro y seguro para hablar de regla gramatical es su imposibilidad de justificación mediante una realidad (empírica o platónica) independiente de la regla. Otro ejemplo muy claro con respecto a dicha imposibilidad lo encontramos en Philosophical Grammar (Wittgenstein [1974] 1992, § 133), cuando Wittgenstein indica que las reglas gramaticales son arbitrarias, al igual que una unidad de medida, en el sentido de que la elección de una unidad de medida (que no es más que un sistema de reglas del tipo ' $1 \mathrm{~m}=1000 \mathrm{~mm}$ ') es independiente de las propiedades físicas del objeto de la medida. Por tanto, la gramática es a priori (Wittgenstein [1975a] 1997, § 1), y lo es precisamente porque no hay una realidad independiente que pueda servir para justificar la gramática, pues sin ella, las palabras para los colores, por ejemplo, no tendrían aún significado. Toda descripción y relación entre colores presupone ya la gramática.

Volviendo a la matemática, el propio Wittgenstein (1975b, p. 48) indica que expresiones matemáticas como ' $2+2=4$ ' son reglas. Concretamente, son reglas que en enunciados empíricos permiten poner ' $2+2$ ' en lugar de '4' (cf. Wittgenstein 1975b, p. 82). No hay duda, por tanto, de que las ecuaciones matemáticas son reglas gramaticales, dado que el intercambio entre expresiones no depende de las propiedades de los objetos que aparecen en enunciados empíricos del tipo 'dos piedras + dos piedras $=$ cuatro piedras'. No se puede acudir a una realidad independiente de la regla, como pueda ser la realidad empírica (obviamente, tampoco sería adecuado acudir a una realidad platónica) para justificar la regla, pues el intercambio entre expresiones presupone ya la regla. ${ }^{8}$ Por tanto, ver que '2+2' es intercambiable con '4' no depende de ninguna comparación con los hechos; siendo esto último algo que Wittgenstein ya presentó en el Tractatus (véase 6.2321).

A modo de conclusión, la posición de Wittgenstein a lo largo de su obra es eminentemente antidescriptivista, no sólo anti-platonista, una descripción que se queda corta a la luz de lo aquí expuesto. La noción de objeto lógico-matemático es una noción puramente formal. En su etapa posterior, Wittgenstein dejó claro que las reglas que constituyen tales objetos lógico-matemáticos (como los sistemas formales o procedimientos mecánicos finitos que generan las proposiciones o los números naturales) son reglas gramaticales que no rinden cuentas ante ninguna realidad. Esta perspectiva se puede aplicar al antecedente histórico del convencionalismo, el formalismo, tal y como veremos a continuación.

\section{III}

Como he indicado en la $\S$ I. el formalismo es una concepción de la lógica y de la matemática cuyo principal iniciador fue Hilbert. ${ }^{9}$ A comienzos del siglo XX. Hilbert propuso el famoso programa finitista por medio del cual pretendía probar la consistencia de la aritmética empleando métodos finitistas; esto es, que no pueda darse el caso de que una proposición A y su negación no A sean ambas teoremas. Asimismo, el segundo programa de Hilbert estaba relacionado con el llamado 'problema de la decisión' (Entscheidungsproblem). Tal problema consistía en establecer un procedimiento de decisión (Entscheidungsverfahren) que permitiera determinar o decidir, en un número finito de operaciones, si

\footnotetext{
${ }^{8}$ Este intercambio o substitución entre expresiones es "el método que utiliza la matemática para llegar a sus ecuaciones [...]"; "las ecuaciones expresan la sustitutibilidad de dos expresiones; y pasamos de cierto número de ecuaciones a ecuaciones nuevas al substituir unas expresiones por otras de una manera que responda a las ecuaciones" (Wittgenstein 1922, 6.24).

9 Wittgenstein ([1974] 1992, [1975a] 1997) también se mostró crítico con respecto a la propuesta de Hilbert denominada 'metamatemática', considerada ésta como una metateoría de la matemática; esto es, primero se formalizaba la teoría matemática que se quería estudiar para después considerar tal teoría el objeto de un estudio matemático. Para Wittgenstein, lo que Hilbert ofrecía era un cálculo como cualquier otro. Es decir, no ofrece un meta-cálculo, sino un cálculo.
} 
una expresión o fórmula bien formada es o no válida. Es bien conocido que tales esfuerzos fueron cercenados por los trabajos de Gödel (1931), Church (1936), Turing (1937) y Post (1943).

Estos autores trataron de caracterizar formalmente la noción de procedimiento mecánico finito (esto es, de algoritmo) y resolver así el problema de la decisión, presentando diferentes respuestas consistentes en sistemas formales tales que por medio de un conjunto de reglas y dado un input, obtuvieran un output, valor o resultado, a través de un conjunto finito de pasos.

Entre estos intentos, podemos destacar, como respuesta directa o indirecta a dicho problema: (a) las funciones recursivas primitivas (Skolem 1923, Gödel 1931) ${ }^{10}$, generales (Gödel 1934) ${ }^{11}$ y parciales (Kleene 1938, 1943, 1952); ${ }^{12}$ Las diferentes clases de funciones recursivas trataron de capturar la clase de las funciones efectivamente computables, para las que existía un procedimiento efectivo de cálculo. ${ }^{13}$ En este sentido, la recursión mostró ser un método por medio del cual las funciones podían ser definidas y, a partir de ahí, efectivamente calculadas.

(b) Turing también formalizó la noción de computación mediante el formalismo conocido como la máquina de Turing (1937). En su caso, la Tesis de Turing, asevera que una función es computable si y sólo si es computable por una máquina de Turing (Soare 2009, p. 373). Turing (1937) presentó un sistema formal que consistía en una máquina de carácter abstracto equipada con cinta potencialmente infinita dividida en cuadrados, cada uno de los cuales podía expresar un símbolo. Asimismo, la máquina cuenta con una cabeza lectora que escanea un único cuadrado de la cinta cada vez, con una serie finita de condiciones $\left(q_{1} \ldots q_{\mathrm{n}}\right)$, que son las distintas configuraciones de la máquina; una serie de símbolos (por ejemplo, 0 y 1); y un conjunto de operaciones (como por ejemplo, escribir un nuevo símbolo; borrar un símbolo escrito; o mover su cabeza lectora un cuadrado hacia la izquierda o hacia la derecha). ${ }^{14}$

(c) Por su parte, Post $(1921,1943,1944)$ definió un formalismo que consiste en un sistema capaz de generar todas las proposiciones de la lógica de enunciados, y, de ahí, todas las tautologías. Así, sea $g$ un enunciado de la lógica proposicional y $P$ una variable operacional aplicada a dicho enunciado, el sistema produce un enunciado g' que sustituye a $g$. Esto se expresa en su sistema de producción normal como sigue: $g P \rightarrow P g$ ' (Post 1943, p. 199). ${ }^{15}$ Este procedimiento de decisión permite decidir (al igual que ${ }_{10}$ Un ejemplo de función recursiva primitiva (adición): $a+0=a$, [si se parte desde ' 1 ', $a+1=a^{\prime}$ ] Def.; $a^{+}(b+1)=(a+b)+1$ Def.
${ }^{11}$ Un ejemplo de función recursiva general: $\varphi(0, y)=\psi(y)$ Def., $\varphi(x+1,0)=\chi(x)$ Def., $\varphi(x+1, y+1)=\varphi(x, \varphi(x+1, y))$ Def. Esta clase incluye las
primitivas, pues estas últimas no son las únicas funciones totales que pueden definirse recursivamente. La diferencia fundamental con
respecto a las primitivas es que la recursión se aplica sobre, al menos, dos variables simultáneamente (véase Gödel 1934, p. 69).
${ }^{2}$ Una clase que recibe su nombre del hecho de que una función no necesita estar definida para todas las n-tuplas de números naturales
que toma como argumentos y que incluyen a las funciones recursivas primitivas y a las funciones recursivas generales como aquellas
funciones parciales definidas para todas las n-tuplas de números naturales que toma como argumentos. Tal clase es extensionalmente
equivalente al formalismo de Turing, lo que me permitió definir la Tesis de Turing-Kleene ("una función es computable si y sólo si es
computable por una función recursiva parcial o, de forma equivalente, por una máquina de Turing" y la Tesis de Kleene, incluida en la
anterior (Mota 2013, \$2). La primera muy bien podría sustituir a la Tesis de Church-Turing, dados los errores cometidos por Church
(Soare, 2009).

${ }^{13}$ No confundir el hecho de que se formularan diferentes CLASES de funciones recursivas con DISTINTOS significados de recursión, como parece hacer Tomalin (2011), que propone tantos significados de recursión como clases hay -ignorando la clase de las funciones recursivas parciales- lo cual es un error. El significado de 'recursión' es el mismo, sólo cambia el alcance de la clase; todas ellas se definen por medio de los esquemas I-V (junto con el esquema VI en el caso de las clases de las generales y las parciales) y, por tanto, haciendo uso de la definición por inducción o por recursión (esquema V) (cf. Kleene 1943, 1952). Nótese, por otro lado, que la inducción también puede ser expresada por medio de la iteración. Así, siguiendo a Wittgenstein ([1974] 1992, p. 32), éste nos dice que es claro que debe haber una "prueba" iterativa que "transmita la idea de que así debe ocurrir con todos los números"; esto es, una regla que concuerda con la inducción: $f(1)=a+b ; f(1) \times(a+b)=(a+b)^{2}=f(2) ;(a+b)^{n}=f(n) ; f(n) \times(a+b)=f(n+1)$. Así "una vez que se tiene la inducción, todo ha terminado" (Wittgenstein [1974] 1992, § 32).

${ }^{14}$ Así, es frecuente expresar las instrucciones de la máquina como sigue: $\left[\mathrm{E}_{0}, 0, \mathrm{E}_{1}, 1\right]$. Esta cuádrupla expresa que la máquina está en el estado $E_{0}$, escaneando el símbolo ' 0 ' y pasa al estado $E_{1}$ al sustituir ' 0 ' por ' 1 '. Esta manera de proceder es iterativa dado que se llevan a cabo diferentes operaciones de manera sucesiva, pasando de una configuración a otra (esto es, de un estado a otro), pero es posible definir recursivamente cada término generado. Además, aunque el formalismo de Turing es considerado como un modelo de computabilidad, esto no niega que se pueda concebir como una adecuada formulación de un sistema formal o, también, de un procedimiento mecánico finito (véase Gödel 1964, pp. 71-72).

15 Otras formulaciones también fueron relevantes: véase Marion (1998), Odifreddi (2001) y Frascolla (1994) para diferentes análisis de las relaciones entre las propuestas de Church $(1932,1936)$ y su cálculo- $\lambda$, por un lado, y las propuestas de Wittgenstein en el Tractatus, por otro. También recomiendo Rodych (1999, 2002, 2003), Wrigley (1977) y Shanker (1987), que muestran el conocimiento que Wittgenstein tenía sobre los avances en la Teoría de la computabilidad. 
el de Wittgenstein) en un número finito de pasos si una proposición es o no una verdad lógica (alias "proposición de la lógica" o "tautología").

Wittgenstein, en su Philosophical Remarks ([1975a] 1997, § 154), hace una observación de gran interés y pertinencia en este punto. Un sistema formal, como lo son todos y cada uno de los sistemas que he mencionado, es una serie de formas (esto es, una serie ordenada por relaciones internas (4.1252)). ${ }^{16} \mathrm{Si}$ son series de formas, entonces caen bajo la forma general de una serie de formas, que en este caso, podemos formular como la forma general de un procedimiento mecánico finito (lo cual no expresa sino una variable): $[\tilde{\sigma}, \tilde{\varepsilon}, \tilde{O}(\tilde{\varepsilon})]($ Mota 2013, 2014). Esta expresión recoge lo que es esencial a todo procedimiento mecánico efectivo, esto es, lo que todos ellos tienen en común. ${ }^{17}$ En relación con los que he mencionado, $\tilde{\sigma}$ estaría por un conjunto de ítems iniciales, tales como números naturales, proposiciones elementales, axiomas; $\tilde{\varepsilon}$ sería un subconjunto de tales ítems iniciales y $\tilde{O}(\tilde{\varepsilon})$ sería un término generado a partir de un elemento previamente computado $\tilde{\varepsilon}$ al que se le aplica la variable operacional $\tilde{O(}$ ), cuyos valores pueden ser las operaciones de la aritmética, las operaciones lógicas o las operaciones que realiza una máquina de Turing, por poner unos ejemplos. Así, para todos ellos es

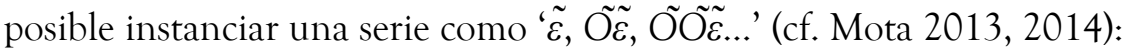

(a) En el caso de una función recursiva

$$
\begin{aligned}
& \tilde{\varepsilon}, \tilde{O} \tilde{\varepsilon}, \tilde{O O} \tilde{\varepsilon}, \ldots \\
& (2+2),(2+2)+1,((2+2)+1)+1, \ldots \\
& (2+2),(2+3),(2+4), \ldots
\end{aligned}
$$

Tenemos pues una serie de definiciones del tipo

$2+4=(2+3)+1$

(b) En el caso del formalismo de Post (muy parecido al de Wittgenstein)

$$
\begin{aligned}
& \tilde{\varepsilon}, \tilde{\mathrm{O} \tilde{\varepsilon}}, \tilde{\mathrm{O} O \tilde{\varepsilon}}, \ldots \\
& g, \operatorname{Pg}, \operatorname{PPg}, \ldots
\end{aligned}
$$

que en el caso de Wittgenstein sería

$$
\tilde{\xi}, N^{\prime}(\tilde{\xi}), N^{\prime} N(\tilde{\xi}), \ldots
$$

Esta serie de definiciones la podemos formular como sigue (sólo muestro el paso recursivo):

$$
P^{(n)} \cdot(g)=P^{\prime} P^{(n-1)} \cdot(g) \cdot / N^{(n)} \cdot(\tilde{\xi})=N^{\prime} N^{(n-1)} \cdot(\tilde{\xi}) .
$$

(c) En el caso del formalismo de Turing

$$
\begin{aligned}
& \tilde{\varepsilon}, \tilde{O} \tilde{\varepsilon}, \tilde{O O} \tilde{\varepsilon}, \ldots \\
& \mathrm{E}_{0}, \tilde{O}\left(\mathrm{E}_{0}\right), \tilde{O} \tilde{O}\left(\mathrm{E}_{0}\right), \ldots
\end{aligned}
$$

que en el caso de la generación de los números naturales se puede escribir:

$\mathrm{E}_{0}$; SubO/1' Esc0' ( $\left.\mathrm{E}_{0}\right)$; >' Esc1' (Sub0/1' EscO' $\left.\left(\mathrm{E}_{0}\right)\right),{ }^{18} \ldots$ que significa:

$\mathrm{E}_{0}, \mathrm{E}_{1}\left(=\mathrm{Sub0} / 1^{\prime} \mathrm{EscO}^{\prime}\left(\mathrm{E}_{0}\right)\right), \mathrm{E}_{2}\left(=>^{\prime} \mathrm{Esc1}^{\prime}\left(\mathrm{Sub0} / 1^{\prime} \mathrm{Esc0} \mathrm{O}^{\prime}\left(\mathrm{E}_{0}\right)\right)\right), \ldots$ y así sucesivamente. ${ }^{19}$

Por tanto, cada estado/configuración o término se puede definir como sigue:

$$
\left.\tilde{O}^{(n)}(\tilde{\varepsilon})=\tilde{O^{\prime}} \tilde{O}^{(n-1)}\right)(\tilde{\varepsilon}) \text {. }
$$

\footnotetext{
16 Por otro lado, como Wittgenstein (1922) indica en 5.232, "[l]a relación interna que ordena una serie equivale a la operación mediante la que un término resulta a partir de otro".

17 De manera análoga a la forma general de una proposición, que recoge lo que es esencial a toda proposición, lo que todas ellas tienen en común $(1922,5.471)$.

18 'EscX' significa 'escanear X'; 'SubX/Y' significa 'sustituir X por Y'; '>' significa 'mover a la derecha'.

19 Por ejemplo, $\mathrm{E}_{1}$ es el estado de la máquina obtenido estando previamente en $\mathrm{E}_{0}$, escaneando 0 , y substituyendo 0 por 1.
} 
Así, la expresión 'Sub0/1' Esc0'>' Esc1' (Sub0/1' Esc0' $\left.\left(\mathrm{E}_{0}\right)\right)$ ' muestra que, estando en $\mathrm{E}_{0}(=0)$, se aplican las operaciones 'Esc0' y 'Sub0/1', lo que permite calcular '1', pasando así al estado $\mathrm{E}_{1}$. Mediante la aplicación de las operaciones 'Esc1', '>', 'Esc0' y 'Sub0/1' la máquina calcula '2' y así sucesivamente. La recursión caracteriza la definición de los $n$ estados/configuraciones, términos o formas (v.gr., ' $\left.\tilde{O}^{(n)}(\tilde{\varepsilon})^{\prime}\right)$ efectivamente computados por una máquina de Turing. Esto caracteriza qué hace una máquina de Turing (lo que se puede aplicar al resto de formalismos), esto es, generar recursivamente una serie de términos, pero cómo lo hace queda descrito por medio de la iteración, esto es, por medio de la repetición sucesiva de operaciones sobre el último resultado calculado (cf. Mota 2013, 2014). Por tanto, para todos ellos es posible formular la forma general de un procedimiento mecánico finito por medio del siguiente sistema de reglas (Mota 2013, 2014):

$$
\begin{aligned}
& \tilde{O}^{(0)},(\tilde{\varepsilon})=\tilde{\varepsilon} \text { Def., } \\
& \tilde{O}^{(n)}(\tilde{\varepsilon})=\tilde{O^{\prime}} \tilde{O}^{(n-1)}(\tilde{\varepsilon}) \text { Def. }
\end{aligned}
$$

En Mota $(2013,2014)$ mostré que la forma general, que es un concepto formal, muestra lo que todos ellos tienen en común. Por tanto, todos estos procedimientos mecánicos efectivos de decisión son instancias, objetos matemáticos, que caen bajo dicha forma general. ${ }^{20}$ Tales objetos matemáticos son sistemas formales, cálculos, construidos y constituidos por reglas gramaticales. ${ }^{21}$

Por tanto, desde la perspectiva de Wittgenstein, tenemos que deshacer la imagen de la matemática como ciencia cuyas expresiones describen (y descubren) objetos matemáticos y hechos acerca de éstos, como puedan ser, series o procedimientos mecánicos finitos. Todos estos objetos matemáticos formales, o sistemas formales, son invenciones o construcciones matemáticas, constituidos por reglas gramaticales. Las ecuaciones matemáticas no son proposiciones susceptibles de verificación o falsación en contraste con una realidad empírica o platónica, como defienden los descriptivistas. Son reglas gramaticales que fijan el significado de ciertos signos (no aseveran o niegan nada, no son una figura de ninguna realidad). Estar en desacuerdo con una regla como ' $2+2=4$ ' (la cual muestra que podemos substituir ' $2+2$ ' por ' 4 ' constituyendo, de este modo, el significado de ambas expresiones) no es negar una regularidad que está de acuerdo con los hechos; es o bien no comprender los significados de ' $2+2$ ' y de '4', o bien atribuir otro significado (o ninguno) a tales expresiones.

\section{IV}

En éste último apartado, voy a comentar brevemente algunas cuestiones que se desprenden del análisis presentado en las secciones anteriores y a explicar cómo se aplican a propuestas que, haciendo uso de cálculos, conciben los dominios cognitivos como órganos mentales (Chomsky 2007a). Centraré mi análisis en el programa chomskiano. En concreto, analizaré el procedimiento generativo/computacional en términos de un formalismo y la noción de representación mental como un concepto formal, interno a la notación conceptual del sistema, del cálculo, y defenderé que no comporta ninguna carga ontológica. En este sentido, no son representaciones de nada.

\footnotetext{
${ }^{20}$ Las diferencias intensionales (esto es, cómo proceden u operan) son importantes para ver, por ejemplo, qué formulación puede ser más clara o perspicua, pero no es esencial a la formulación de la forma general de un procedimiento mecánico finito, entendido como concepto formal. Así, no es esencial que se implemente recursivamente (como las funciones recursivas) o iterativamente (como la máquina de Turing; siendo posible tanto definir cómo implementar iterativamente una función definida por recursión; véase Robinson 1947, Abelson et al. 1996). Del mismo modo que para la formulación de la forma general de una proposición no son esenciales las diferencias intensionales entre proposiciones. La razón es que la forma general expresa lo que todos ellos tienen en común (Mota 2013, 2014). Por otro lado, el 'último' Wittgenstein enfatiza que los procedimientos muestran los llamados 'parecidos de familia', una propuesta más anti-esencialista que la de la primera etapa. En todo caso, me parece muy relevante y acertada la apreciación de que los procedimientos mecánicos finitos son después de todo, series de formas.

${ }^{21}$ Una definición recursiva es una regla gramatical que, como Wittgenstein ([1975a] 1997, §163) señala en Philosophical Remarks, es una regla fundamental del sistema que nos indica cómo puedo proceder (y como tal, no se puede aseverar o negar). Del mismo modo, en Philosophical Grammar ([1974] 1992), nos indica que "es una regla para la construcción de reglas de sustitución, o también el término general de una serie de definiciones [formas]" (36, p. 851).
} 
Desde sus primeros escritos, Chomsky $(1959$, p. 143) ha considerado que, desde un punto de vista conceptual, la teoría de la gramática puede verse como el estudio de una clase especial de funciones recursivas. Esta idea persiste de una u otra manera en diferentes trabajos, como por ejemplo, en The Logical Structure of Linguistic Theory (Chomsky 1975), en el que reconoce la importancia de los avances de la Teoría de la Computabilidad para el estudio del lenguaje natural, o, más recientemente, en Chomsky (2007b), donde indica que uno de los principales factores que han impulsado el desarrollo de la perspectiva biolingüística ha sido el trabajo realizado dentro de la Teoría de la Computabilidad, que ha permitido estudiar más seriamente los mecanismos formales de la gramática generativa. En esta misma línea, Chomsky (2012) ha indicado que el estudio del lenguaje-I, entendido como un sistema dotado de infinitud discreta, cae dentro de la Teoría de la Computabilidad, siendo, de este modo, visto como un procedimiento computacional que genera recursivamente una cantidad potencialmente infinita de expresiones jerárquicamente estructuradas, interpretadas por los sistemas sensorio-motor y conceptual-intencional (Chomsky 1995a p. 226). Tal procedimiento está basado en Merge.

La forma general de Merge se puede formular de la siguiente manera, siguiendo la notación de Wittgenstein: $\left[\mathrm{N}, \mathrm{O}_{s}, \mathrm{M}\left(\mathrm{O}_{s}\right)\right]$. Así, $\mathrm{N}$ hace referencia a ítems léxicos y objetos sintácticos que configuran la numeración, $\mathrm{O}_{s}$ hace referencia a un objeto sintáctico cualquiera de la serie generada y $\mathrm{M}\left(\mathrm{O}_{s}\right)$ hace referencia a un objeto sintáctico nuevo generado a partir de $\mathrm{O}_{s}$ mediante la aplicación de $\mathrm{M}\left(\right.$ ) ${ }^{22}$ Así, y de manera esquemática, vemos que lo que Merge hace (esto es, generar/definir recursivamente objetos sintácticos, independientemente de su estructura interna, como vemos en la definición formal) queda expresado mediante la serie ' $\mathrm{O}_{s}, \mathrm{M}^{\prime}\left(\mathrm{O}_{\mathrm{s}}\right), \mathrm{M}^{\prime} \mathrm{M}$ ' $\left(\mathrm{O}_{\mathrm{s}}\right), \ldots .{ }^{23}$ Esto puede definirse recursivamente de manera formal como sigue (Mota 2013, nota 2, 2014):

$$
\begin{aligned}
& M^{(0) '}\left(O_{s}\right)=O_{s}, \\
& M^{(n)}\left(O_{s}\right)=M^{\prime} M^{(n-1)}\left(O_{s}\right) .
\end{aligned}
$$

Por otra parte, Chomsky (2007a, p. 6, 2008, p. 139) ha insistido en que el modo de operar (o de implementarse de manera abstracta) de este procedimiento generativo (esto es, cómo procede) es aplicando iterativamente la operación Merge. Así, la aplicación iterativa queda descrita mediante la siguiente serie, que muestra, en cada paso, las $n$ veces que Merge se aplica de forma sucesiva, lo cual puede generar todo tipo de expresiones (con auto-inclusión o no) del lenguaje natural: $\mathrm{M}^{(0)}, \mathrm{M}^{(1)}$, $M^{(2)}, \ldots, M^{(n)}, \ldots, M^{(n+1)}$; esto es: $M^{\prime} M^{(n)}\left(O_{s}\right)=M^{(n+1)},\left(O_{s}\right)$.

Atendiendo a la definición formal, salta a la vista que el procedimiento mecánico finito propuesto por Chomsky es una instancia de la forma general de una serie de formas, pues una serie de objetos sintácticos es una serie ordenada por relaciones internas. De este modo cae bajo la forma general arriba presentada. $^{24}$

Pero, además de su carácter abstracto, Chomsky (2007a, p. 8, 2010, p. 53), establece que Merge es una propiedad del lenguaje genéticamente determinada (2007a p. 8, 2010, p. 53). Así, Chomsky va más allá de la Teoría de la Computabilidad al hablar de la evolución del lenguaje y especular con que el cerebro sufrió una reorganización, quizá por algún evento genético, y ello propició la aparición de Merge (Chomsky 2006, p. 184, 2010, pp. 58-59). En este sentido, y partiendo de la idea de que la facultad del lenguaje es un sistema biológico, Chomsky (2005) formula tres factores que se deben tener en cuenta en el desarrollo de la facultad del lenguaje: un primer factor que correspondería con la

\footnotetext{
22 Sea la numeración $N=\{$ Juan, canta, muchas, canciones $\}$, lo que Merge hace es tomar dos ítems léxicos (definida aquí como una operación binaria) 'muchas' y 'canciones' y forma el objeto sintáctico $\{$ muchas, canciones $\}=\{X, Y\}$. Mediante otra aplicación, Merge genera un nuevo objeto sintáctico \{canta, \{muchas, canciones\}\}, formado a partir de un objeto sintáctico previamente computado (\{muchas, canciones\}); por último, Merge genera $\{$ Juan, $\{$ canta, $\{$ muchas, canciones $\}\}\}$.

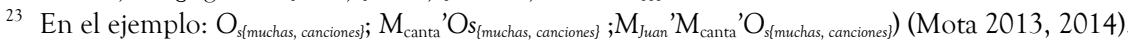

24 En una correspondencia personal con Chomsky en la que le planteé esta cuestión, respondió que no dudaba de que Merge podía formularse fácilmente dentro de un concepto general de procedimiento mecánico finito. Expuso que, entendiendo Merge como una operación binaria, tenemos dos posibilidades lógicas: Merge Externo ( $X$ distinto de $Y$ ) y Merge Interno ( $X$ es un término de $Y$ ). Esto es absolutamente válido. Sin embargo, la forma general de Merge arriba mostrada ya incluye tales posibilidades y no es necesario expresar tales posibilidades de manera explícita, pues $M(O s)$ tiene la misma forma general $O s=\chi, Y$, tanto si $\chi$ e $Y$ son distintos como si $\chi$ es un término de $Y$.
} 
dotación genética (la Gramática Universal), un segundo factor que hace referencia a la experiencia y un tercer factor que incluye principios no específicos de la facultad del lenguaje.

Dentro del tercer factor, se incluye, entre otros, un subtipo que hace referencia a la eficiencia computacional. Un ejemplo de eficiencia computacional sería la condición de la no-injerencia (no tampering), esto es, tomando $X$ e $Y$, Merge genera $\{X, Y\}=Z$, dejando $X$ e $Y$ sin modificaciones, sin añadir o quitar elementos (Chomsky 2010, p. 52). Sin embargo, las condiciones de eficiencia computacional sólo definen el cálculo, el sistema formal. Chomsky, en mi opinión, parece mezclar distintos niveles de análisis. Sin duda, la capacidad/facultad del lenguaje puede entenderse como un objeto natural, susceptible de investigación empírica, pero no así el procedimiento mecánico finito construido para su caracterización abstracta, pues éste no es más que un cálculo, susceptible de una investigación formal (matemática). Las propiedades del cálculo (como la recursión) están determinadas a priori por las reglas que lo constituyen (cf. Mota 2013, 2014).

Anthony Kenny $(1989,1990)$ expuso esta cuestión de una manera clara, al proponer que la mente es "la capacidad de adquirir habilidades intelectuales" (Kenny 1990, p. 204). En este sentido, hay que distinguir entre poseedores y vehículos. El poseedor de la habilidad lingüística es un ser humano y el vehículo "es la parte de su poseedor por virtud de la cual éste es capaz de ejercer la habilidad...algo concreto y más o menos tangible" (Kenny 1990, p. 205). Podemos hacer una distinción entre las personas (el poseedor), la mente (conjunto de capacidades) y el cerebro (el vehículo de tales capacidades). Evidentemente, las personas y sus cerebros son objetos físicos, pero no así la mente, que es un conjunto de capacidades. Conviene tener presente también que tal distinción no es una distinción metafísica/ontológica, sino conceptual, en virtud de la cual se definen 'mente' y 'vehículo'. En este sentido, Kenny propone que pensemos en una calculadora como analogía a lo que se acaba de exponer: el fisiólogo estaría a la par junto con el ingeniero electrónico, mientras que el psicólogo está en una posición análoga a la del matemático, pues intenta descubrir el algoritmo que usa la calculadora (1990, p. 206). La calculadora tiene la habilidad de computar un algoritmo y su análisis exige una investigación matemática, más que electrónica. El aparataje electrónico sería el vehículo.

Sin embargo, si atendemos al naturalismo metodológico que defiende Chomsky, el lenguaje, entendido como un órgano mental o un sistema biológico, ha de ser considerado como un objeto real, esto es, como el resto de objetos del mundo, y su estudio ha de ser abordado desde una perspectiva naturalista, esto es, como las ciencias naturales estudian otros objetos del mundo. Parecería, por tanto, que esto resulta incompatible con el anti-realismo que vengo exponiendo a lo largo de este artículo. Profundicemos más en esta cuestión. Bajo el naturalismo metodológico, tal y como acabo de exponer, Chomsky hace una aproximación a la mente bajo la cual considera el lenguaje como un elemento del mundo natural (Chomsky 1995b, p. 1). Chomsky subraya aquí que usa el término 'mente' y 'mental' sin ninguna implicación metafísica, estando a la par de términos como 'químico', 'óptico' o 'eléctrico' (Chomsky 1995b, p. 1). Tales términos seleccionan ciertos aspectos del mundo como foco de investigación, entendiendo por 'mente' los aspectos mentales del mundo (Chomsky 1995b, p. 1). Desde la perspectiva biolingüística, nos dice Chomsky (2006), el lenguaje es un componente de la mente, entendiendo 'mente' en el sentido de los científicos del siglo XVIII, de tal manera que, no siendo posible postular un problema mente/cuerpo coherente, sólo podemos considerar los aspectos del mundo denominados mentales como el resultado de una organización orgánica tal como la del cerebro (Chomsky 2006, p. 173, véase también 2005, p. 2).

Estas consideraciones generales han sido algo más precisadas en un interesante trabajo reciente. En dicho trabajo, Berwick, Friederici, Chomsky \& Bolhuis (2013, p. 90), nos indican que el lenguaje humano está asentado sobre un mecanismo computacional particular realizado neurológicamente. En este primer apunte, es interesante notar que el mecanismo computacional no sólo es una herramienta construida para caracterizar el lenguaje de manera abstracta, sino que se postula que tal procedimiento mecánico finito está realizado neurológicamente. Es decir, tal procedimiento está a la par que, por ejemplo, las neuronas; son objetos del mundo. Por decirlo de otra manera, dicho procedimiento no sólo es una clase especial de funciones recursivas (ver supra), sino que también es un objeto del mundo. 
Como señalé unas líneas más arriba, el procedimiento mecánico finito basado en Merge genera objetos sintácticos (de los cuales me ocuparé más abajo), que son interpretados por dos sistemas, el sensorio-motor (encargado entre otras cosas de la externalización) y el intencional-conceptual (que hace referencia, entre otras cuestiones, al pensamiento y a la planificación de la acción) (Chomsky 2010, p. 269). Obviamente, en el plano neurológico, tal mecanismo generativo/computacional debe diferenciarse de ambos sistemas de interfaz. Berwick et al. (2013, p. 93) presentan diferentes regiones identificadas tanto con el mecanismo computacional como con los sistemas de interfaz. Así, dicho mecanismo está sustentado (o realizado neurológicamente) por el área de Brodmann 44 y el córtex temporal superior posterior. El sistema sensorio-motor, por su parte, estaría sustentado por el córtex premotor y el córtex temporal superior. Finalmente, el procesamiento semántico (propio del sistema conceptual-intencional) está sustentado por el área de Brodmann 45 y por el córtex frontal inferior, así como por porciones del córtex temporal. Así, nos dicen Berwick et al. (2013, p. 93), el estudio de las bases neurológicas del lenguaje debe considerar aquellas partes o regiones particulares del cerebro que representan el mecanismo computacional así como aquellos sistemas de interfaz. Por tanto, cada uno de estos sistemas consisten en las mencionadas regiones particulares del cerebro, conectadas vía tractos específicos (véase Berwick et al. 2013 para más detalles).

Sin embargo, creo que tal estudio, aunque muy interesante y desde luego legítimo en relación con la facultad del lenguaje, no es un obstáculo para el anti-realismo que vengo exponiendo. El estudio del lenguaje en este nivel neurológico es, siguiendo a Kenny, el estudio del vehículo, del cerebro. El mecanismo computacional (cuyo estudio apropiado es una investigación matemática) no consiste en estas o aquellas regiones cerebrales. Tal procedimiento es una construcción matemática que no supone el descubrimiento de ningún objeto mental del mundo natural. El estudio del cerebro, por su parte, sí es el estudio de un objeto del mundo natural, susceptible de una metodología naturalista. Eso sí, el estudio se realiza en otro nivel de análisis. De manera análoga, Alemán (2011) indica, en su análisis introductorio a una primera versión del argumento de indispensabilidad, que:

[P] uesto que una ciencia como la física describe y predice con éxito lo que acontece en la realidad circundante, tal teoría tiene que ser verdadera y si esto es así, entonces tienen que existir las entidades a las que se refieren los términos de la teoría (Alemán 2011, p. 23).

Así, continúa Alemán (2011, p. 23), no sólo tienen que existir los electrones y los quarks, sino que también tienen que existir los números, funciones, [...], referidos por los términos matemáticos empleados en la formulación de tal teoría física. Así, una cosa es que construyamos un sistema formal, un cálculo, y llevemos a cabo un análisis matemático del mismo, y otra cosa completamente diferente es que (I) lo postulemos como un objeto de una realidad (empírica o platónica), y (II) entendamos que ambos niveles de análisis, a saber, el lógico-matemático y el empírico en este caso, no sólo están al mismo nivel, sino que son el mismo; esto es, las entidades físicas y matemáticas existen en la misma realidad espacio-temporal y las abordamos mediante una metodología naturalista. Esta concepción realista del mecanismo formal propuesto por Chomsky parece estar detrás de la idea de que dicho mecanismo es un objeto del mundo natural.

Sin embargo, una cosa es decir que el lenguaje es un sistema biológico y otra muy diferente decir que la caracterización abstracta hecha mediante la formalización planteada por Chomsky sea un objeto real del mundo natural; es decir, que tal formalización describa un objeto del mundo natural. Tal formalismo es, en cierto sentido, autónomo con respecto a cualquier realidad en el sentido de que no es descriptivo de ella. En este sentido, en el momento en el que se construye un formalismo se están constituyendo tanto los signos lógico-matemáticos empleados en éste como las reglas para su uso dentro del sistema (por ejemplo, un sistema formal o procedimiento mecánico finito, puede calcular en un número finito de pasos, definidos unos axiomas, otras verdades lógicas mediante la aplicación de operaciones siguiendo un conjunto de reglas, pudiendo ordenarse tales verdades en series; cf. supra). De este modo, no se descubren los objetos mentales como el mecanismo generativo/computacional propuesto por Chomsky, sino que se crean, se construyen y se constituyen mediante las reglas gramaticales. 
Relacionado con esto último, conviene recordar que una regla gramatical no niega ni asevera nada y por tanto no describe ninguna realidad. Así habría que distinguir entre (I) la expresión " $2+2$ " se puede intercambiar con la expresión " $(2+1)+1$ ”, (II) el sujeto $\mathrm{S}$ usa la regla 'R' y (III) el sujeto $\mathrm{S}$ usa la regla ' $R$ ". Así, (I) no es un enunciado empírico y por tanto, las propiedades constituidas por dicha regla gramatical no dependen de ninguna realidad; son autónomas frente a ésta y se constituyen por la regla y, por tanto, dentro del sistema. Por su parte, los enunciados (II) y (III) son enunciados empíricos y, por tanto, lo que se confirma o desconfirma son tales enunciados, no las reglas ' $R$ ' o ' $R$ '. Dichas reglas, así como (I), no dicen nada, no describen ninguna realidad.

A continuación, y para terminar, veremos en qué medida podemos decir que las representaciones mentales son objetos del mundo natural o, por el contrario, constituyen objetos mentales no descubiertos, sino creados mediante la formulación, creación o invención de un formalismo empleado para caracterizar la facultad del lenguaje. Con respecto a la noción de representación mental, Chomsky (1980, p. 5) indica que tal noción caracteriza de manera abstracta las propiedades de ciertos mecanismos físicos, pero que no conllevan ningún importe ontológico; esto es, no es necesario postular la existencia de entidades que pertenezcan a una realidad diferente a la del mundo físico. ${ }^{25}$ En Language and Nature (Chomsky 1995b) ofrece una buena definición de lo que él entiende por representación mental. Así, Chomsky (1995b, p. 52), dice que no hay ninguna cuestión con sentido que tenga que ver con el contenido de una representación interna; ninguna noción como 'contenido' o 'representación de' es considerada dentro de la teoría y, por ello, no tiene sentido plantearse la pregunta sobre su naturaleza. En el caso del estudio del lenguaje, también se habla de representaciones de varios tipos, por ejemplo, representaciones semánticas y fonéticas que son enviadas a otros sistemas como son el sensorio-motor y el conceptual-intencional. Pero tampoco en este caso, insiste Chomsky (1995b, p. 53), tenemos que buscar alguna construcción objetiva de las que sean "representaciones de".

Más recientemente, Berwick et al. (2013, p. 91), recuerdan que el mecanismo computacional formulado para el lenguaje humano incluye una operación, Merge, que construye nuevos elementos representacionales $Z$ desde otros elementos previamente construidos, a saber, $X, Y$. Continúan indicando que una representación interna tal presenta, como señalé más arriba, una versión ordenada para ser externalizada. Por su parte, las computaciones que construyen tanto las representaciones sintácticas por el mecanismo generativo/computacional, como su versión conceptual-intencional (semántica) aluden a lo que Berwick et al. (2013, p. 89), denominan internalización. Relacionado con esto último, Berwick et al. (2013, pp. 93-94) indican que se pueden señalar determinadas regiones cerebrales a modo de correlatos neuronales que sustentan tal construcción de representaciones sintácticas (cf. Berwick et al. 2013, pp. 91-92 para más detalles, por ejemplo, sobre el algoritmo que etiqueta los elementos representacionales). En este sentido, el área de Broca, en concreto, el área de Brodmann 44 (ver supra), se activa durante el procesamiento de estructuras jerárquicas en el lenguaje natural (Berwick et al. 2013, p.94), que también activa el córtex temporal superior posterior, conectados por medio del fascículo arqueado, y partes del fascículo longitudinal superior (Berwick et al. 2013). En todo caso, tal sistema dorsal (que incluye el área Broca, esto es, el área de Brodmann 44) sustenta las computaciones basadas en reglas para la construcción de estructuras jerárquicas. Esto implica cierto realismo sobre las representaciones mentales, tal como Pylyshyn (1991) indicó en un interesante trabajo, ya clásico. Así, las representaciones mentales, como puedan ser las representaciones mentales sintácticas o conceptuales-intencionales, han de estar realizadas neuronalmente y, por ello, no es necesario, como indica Chomsky, postular la existencia de entidades que pertenezcan a una realidad diferente a la del mundo físico. Según esta perspectiva, éstas están, por tanto, explícitamente codificas en el sistema físico, esto es, el cerebro.

Sin embargo, la crítica a este realismo puede hacerse siguiendo la misma crítica presentada unas líneas más arriba, a saber, una cosa es que las construcciones lingüísticas tengan una realización

\footnotetext{
${ }^{25}$ Autores como Fodor (1992) defienden el realismo de las representaciones mentales, entendidas como actitudes proposicionales, indicando que uno es realista en cuanto a las actitudes proposicionales si (a) sostiene que hay estados mentales cuya ocurrencia e interacción causan el comportamiento y (b) sostienen que tales estados mentales son semánticamente evaluables.
} 
neurológica y otra muy diferente es que las denominadas representaciones mentales tengan el mismo estatus ontológico que el de las neuronas. Una vez más, al construir un formalismo que mediante un conjunto de reglas construye objetos sintácticos, se está, de hecho, creando, no descubriendo, tanto las reglas gramaticales como los objetos sintácticos que, siguiéndolas, se constituyen en dicho cálculo. Los objetos sintácticos o representaciones mentales (v.gr. $\{X, Y\}$ o $M^{\prime}\left(O_{s}\right)$ ) son, por tanto, objetos construidos en el momento en el que creamos un formalismo que caracteriza de manera abstracta la facultad del lenguaje. Su aplicación es interna al sistema. De esto se deprende que lo mental no es un mundo independiente del mundo natural, pero tampoco es una parte de éste en el sentido de que es una porción que ha de ser descubierta; es, más bien, un sistema formal creado para caracterizar de manera abstracta determinadas capacidades. Dicha construcción implica la creación de otros "objetos", cuyas propiedades y aplicaciones se constituyen dentro del sistema.

En suma, el aspecto fundamental desde la perspectiva wittgensteiniana que vengo defendiendo es que si concebimos Merge como un procedimiento mecánico finito, entonces hay que entenderlo como una construcción matemática, no como algo determinado genéticamente. Por otro lado, los inputs que Merge toma como argumentos y los valores que, al aplicar una operación a través de una serie de pasos finitos, devuelve, pueden entenderse como representaciones mentales/objetos sintácticos. Pero esto no debe hacer creer que estos objetos existen en una realidad platónica, punto en el que estoy de acuerdo con Chomsky, pero tampoco en una realidad física o empírica, como Chomsky parece sugerir. No son objetos del mundo natural descubiertos, únicamente son construcciones interna al formalismo creado para caracterizar de manera abstracta una capacidad intelectual.

\section{Conclusiones}

En este trabajo he pretendido mostrar que adoptando la perspectiva anti-realista de Wittgenstein, que defendió tanto en el Tractatus como en sus obras intermedias y posteriores, podemos tratar los objetos/sistemas matemáticos formales como invenciones, creaciones o construcciones (Wittgenstein 1978) y no como un sistema de proposiciones que describen una realidad empírica (realismo empírico) o platónica (realismo platónico), sino como un sistema de reglas gramaticales que constituyen el cálculo y el significado (el uso) de los signos y símbolos en él implicados. Un ejemplo de la utilidad de la formulación wittgensteiniana la he mostrado en su aplicación al procedimiento mecánico finito definido por Chomsky, así como a otras nociones (como la de representación mental) implicadas en el mismo.

Bibliografía

Alemán, A. (2011), Lógica, matemáticas y realidad, Madrid: Tecnos.

Berwick, R., Friederici, A.D., Chomsky, N. y J. Bolhuis (2013), "Evolution, Brain, and the Nature of Language”, Trends in Cognitive Science 17: 89-98.

Chomsky, N. (1959), “On Certain Formal Properties of Grammars”, Information and Control 2: 137-167.

Chomsky, N. (1975), The Logical Structure of Syntactic Theory, Nueva York: Plenum Press.

Chomsky, N. (1980), Rules and Representations, Nueva York: Columbia University Press.

Chomsky, N. (1995a), The Minimalist Program, Cambridge, MA: MIT Press.

Chomsky, N. (1995b), "Language and Nature”, Mind 104: 1-61.

Chomsky, N. (2005), “Three Factors in Language Design”, Linguistic Inquiry 36: 1-22.

Chomsky, N. (2006); Language and Mind, Cambridge: Cambridge University Press. 
Chomsky, N. (2007a), “Approaching UG from Below”, en Sauerland, U. y H.M. Gärtner (eds.), Interfaces + Recursion = Language?, Berlín: Mouton, pp. 1-30.

Chomsky, N. (2007b), “Of Minds and Language”, Biolinguistics 1: 9-27.

Chomsky, N. (2008), “On Phases”, en Freidin, R., Otero, C. y M.L. Zubizarreta (eds.), Foundational Issues in Linguistic Theory, Cambridge, MA: MIT Press, pp. 133-166.

Chomsky, N. (2010), “Some Simple Evo Devo Theses: How True Might They Be for Language?", en Larson, R., Déprez, V. y H. Yamakido (eds.), The Evolution of Human Language, Cambridge: Cambridge University Press, pp. 45-62.

Chomsky, N. (2012), “Some Core Contested Concepts”, Proceedings of the CUNY 2012:1-18.

Church, A. (1932), "A Set of Postulates for the Foundation of Logic”, The Annals of Mathematics 33:346-366.

Church, A. (1936), “An Unsolvable Problem of Elementary Number Theory”, en Davis, M. (ed.), The Undecidable, Nueva York: Raven Press, pp. 88-107.

Cutland, N. (1980), Computability: An Introduction to Recursive Function Theory, Cambridge: Cambridge University Press.

Fodor, J. (1992), A Theory of Content and Other Essays, Cambridge, MA: MIT Press.

Frascolla, P. (1994), Wittgenstein's Philosophy of Mathematics, Londres: Routledge.

Frege, G. (1892), “Sobre sentido y referencia”, en Valdés, L. (ed.), Ensayos de semántica y filosofía de la lógica, Madrid: Tecnos, pp. 84-111.

Gödel, K. (1931), “On Formally Undecidable Propositions of the Principia Mathematica and Related Systems I”, en Davis, M. (ed.), The Undecidable, Nueva York: Raven Press, pp. 4-38.

Gödel, K. (1934), “On undecidable propositions of formal mathematical systems”, en Davis, M. (ed.), The Undecidable, Nueva York: Raven Press, pp. 39-74.

Gödel, K. (1944), “La lógica matemática de Russell”, en Mosterín, J. (ed.), Obras completas, Madrid: Alianza, pp. 313. 343.

Gödel, K. (1964), “Postscriptum to Gödel 1931”, en Davis, M. (ed.), The Undecidable, Nueva York: Raven Press, pp. 71 73.

Kenny, A. (1989), The Metaphysics of Mind, Oxford: Oxford University Press.

Kenny, A. (1990), El legado de Wittgenstein, Madrid: Siglo XXI.

Kleene, S.C. (1938), “On Notation for Ordinal Numbers”, The Journal of Symbolic Logic 3: 150-5.

Kleene, S.C. (1943), "Recursive Predicates and Quantifiers”, Transactions of the American Mathematical Society 53: $41-73$.

Kleene, S.C. (1952), Introduction to Metamathematics, Amsterdam: North-Holland Publishing.

Marion, M. (1998), Wittgenstein, Finitism, and the Foundations of Mathematics, Oxford: Oxford University Press.

Monk, R. (1990), Wittgenstein: The Duty of Genius, Nueva York: Free Press.

Mota, S. (2013), “La propiedad de la recursión en el 'Tractatus Logico-Philosophicus' de Wittgenstein y su relación con la Teoría de la Computabilidad y la Lógica Matemática”, Observaciones Filosóficas 17:

http://www.obervacionesfilosoficas.net/lapropiedaddelarecursion.htm

Mota, S. (2014), “La historia y la gramática de la recursión: una precisión desde la obra de Wittgenstein”, Pensamiento y Cultura 17: 20-48.

Mounce, H.O. (1981), Wittgenstein's Tractatus. An Introduction, Oxford: Blackwell.

Odifreddi, P. (2001), "Recursive Functions: An Archaeological Look”, en Claude, C.S., Dinneen, M.J. y S. Sburlan (eds.), Combinatorics, Computability and Logic, Londres: Springer-Verlag, pp. 13-31.

Pinto, S. (2002), "El anti-platonismo del Tractatus de Wittgenstein”, Theoria 13: 137-152.

Post, E. (1921), "Introduction to a General Theory of Elementary Propositions", American Journal of Mathematics 43: 163-185. 
Post, E. (1943), "Formal Reductions of the General Combinatorial Decision Problem", American Journal of Mathematics 65: 197-215.

Post, E. (1944), "Recursively Enumerable Sets of Positive Integers and their Decision Problems", en Davis, M. (ed.), The Undecidable, Nueva York: Raven Press, pp. 305-337.

Pylyshyn, Z.W. (1991), "Rules and Representations: Chomsky and Representational Realism", en Kashir, A. (ed.), The Chomskyan Turn, Oxford: Basil Blackwell, pp. 231-251.

Quine, W.V.O. (1980), From a Logical Point of View, Cambridge, MA: Harvard University Press.

Robinson, R. (1947), "Primitive Recursive Functions", Bulletin of the American Mathematical Society 53: 925-942.

Rodych, V. (1999), “Wittgenstein's Inversion of Gödel's Theorem”, Erkenntnis 51: 173-206.

Rodych, V. (2002), "Wittgenstein on Gödel: The Newly Published Remarks", Erkenntnis 56: 379-397.

Rodych, V. (2003), "Misunderstanding Gödel: New Arguments about Wittgenstein and New Remarks by Wittgenstein", Dialectica 57: 279-313.

Shanker, S.G. (1987), "Wittgenstein versus Turing on Nature of Church's Thesis", Notre Dame Journal of Formal Logic 28: 615-649.

Skolem, T. (1923), "The Foundations of Elementary Arithmetic Established by Means of the Recursive Mode of Thought, without the Use of Apparent Variables Ranging over Infinite Domains", en Van Heijenoort, J. (ed.), From Frege to Gödel. A Source Book in Mathematical Logic, 1879-1931, Cambridge, MA: Harvard University Press, pp. $302-333$.

Soare, R. (1996), "Computability and Recursion”, The Bulletin of Symbolic Logic 2: 284-321.

Soare, R. (2009), "Turing Oracles Machines, Online Computing, and Three Displacements in Computability Theory", Annals of Pure and Applied Logic 160: 368-399.

Tomalin, M. (2011, "Syntactic Structures and Recursive Devices: A Legacy of Imprecision”, Journal of Logic, Language and Information 20: 297-315.

Turing, A. (1937), "On Computable Numbers, with an Application to the Entscheidungsproblem”, en Davis, M. (ed.), The Undecidable, Nueva York: Raven Press, pp. 116-151.

Wittgenstein, L. (1922), Tractatus Logico-Philosophicus, London: Routledge.

Wittgenstein, L. (1958), Philosophical Investigations, Oxford: Blackwell. (Traducción castellana: Investigaciones filosóficas, Barcelona: Crítica, 1988.)

Wittgenstein, L. (1974). Philosophical Grammar, Oxford: Basil Blackwell. (Traducción castellana: Gramática filosófica, México: UNAM, 1992.)

Wittgenstein, L. (1975a). Philosophical Remarks, Oxford: Blackwell. (Traducción castellana: Observaciones filosóficas, México: UNAM, 1997.)

Wittgenstein, L. (1975b), Wittgenstein's Lectures on the Foundations of Mathematics, Cambridge, 1939, Chicago: University of Chicago Press.

Wittgenstein, L. (1978), Remarks on the Foundations of Mathematics, Oxford: Blackwell.

Wrigley, M. (1977), “Wittgenstein's Philosophy of Mathematics”, Philosophical Quarterly 27: 50-59. 